\title{
Birth seasonality in Korean Prader-Willi syndrome with chromosome 15 microdeletion
}

\author{
Aram Yang, MD, \\ Yeon Hee Lee, MD, \\ Soon Young Nam, MD, \\ Yu Ju Jeong, MD, \\ Yechan Kyung, MD, \\ Rimm Huh, MD, \\ Jieun Lee, MD, \\ Younghee Kwun, MD, \\ Sung Yoon Cho, PhD, \\ Dong-Kyu Jin, PhD
}

Department of Pediatics, Samsung Medical Center, Sungkyunkwan University School of Medicine, Seoul, Korea
Purpose: Prader-Willi syndrome (PWS) is a well-known genetic disorder, and microdeletion on chromosome 15 is the most common causal mechanism. Several previous studies have suggested that various environmental factors might be related to the pathogenesis of microdeletion in PWS. In this study, we investigated birth seasonality in Korean PWS.

Methods: A total of 211 PWS patients born from 1980 to 2014 were diagnosed by methylation polymerase chain reaction at Samsung Medical Center. Of the 211 patients, 138 were born from 2000-2013. Among them, the 74 patients of a deletion group and the 22 patients of a maternal uniparental disomy (UPD) group were compared with general populations born from 2000 using the Walter and Elwood method and cosinor analysis.

Results: There was no statistical significance in seasonal variation in births of the total 211 patients with PWS $\left(X^{2}=7.2522, P=0.2982\right)$. However, a significant difference was found in the monthly variation between PWS with the deletion group and the at-risk general population $(P<0.05)$. In the cosinor model, the peak month of birth for PWS patients in the deletion group was January, while the nadir occurred in July, with statistical significance (amplitude=0.23, phase=1.2, low point=7.2). The UPD group showed the peak birth month in spring; however, this result was not statistically significant $\left(X^{2}=3.39, P=0.1836\right)$.

Conclusion: Correlation with birth seasonality was identified in a deletion group of Korean PWS patients. Further studies are required to identify the mechanism related to seasonal effects of environmental factors on microdeletion on chromosome 15.

Keywords: Prader-Willi syndrome, Microdeletion, Birth, Seasonality

\section{Introduction}

Prader-Willi syndrome (PWS) is a genetic disorder caused by a deficiency of imprinted gene expression from the paternal chromosome $15 q 11-15 q 13$. PWS has a prevalence of 1 in 10,000-20,000 individuals. This disease is clinically characterized by neonatal hypotonia, poor feeding in infancy (often associated with failure to thrive), followed by obesity beginning around age $2^{1)}$. Patients with PWS exhibit short stature, cognitive impairment, hypogonadism, hyperphagia, morbid obesity, behavioral problems, and diabetes ${ }^{2}$.

The frequencies of different genetic subtypes in PWS are usually given in the literature as $70 \%$ paternal interstitial deletion of the 15q11-13 region, 25\%-30\% maternal uniparental disomy (mUPD) of chromosome 15, 3\%-5\% imprinting center (IC) defects (microdeletion of PWS-IC, primary epimutation), and unbalanced translocations involving the PWS region ${ }^{3,4)}$.

A group of 97 Korean patients with PWS (median birth year of 2001; interquartile range, 1998-2007; male:female, 58:39) who were diagnosed using methylation analysis were classified in our previous report ${ }^{5)}$ into several detailed genetic subgroups by stepwise genetic analysis: deletions in 66 patients (68\%), mUPD in 28 patients (29.9\%), and primary epimutation in two
Received: 29 September, 2014 Revised: 9 December, 2014 Accepted: 30 December, 2014

Dong-Kyu Jin, MD, PhD

Division of Endocrinology and

Metabolism, Department of

Pediatrics, Samsung Medical

Center, Sungkyunkwan University

School of Medicine, 81 Irwon-ro,

Gangnam-gu, Seoul 135-710, Korea

Tel: +82-2-3410-3525

Fax: +82-2-3410-0043

E-mail: jindk@skku.edu 
patients $(2.1 \%)$. The proportions of genetic subgroups were similar to those given in other literature ${ }^{5)}$.

Consistent with the findings of previous studies, deletion is the most common genetic mechanism in PWS. It has been suggested that the particular genomic architecture of the $15 q 11-q 13$ region, characterized as flanked by low copy repeats, could predispose it to nonallelic homologous recombination $(\mathrm{NAHR})^{6)}$. Molina et al. ${ }^{6)}$ suggested that increased susceptibility of some PWS fathers to generating 15q11-q13 deletions is probably related to the particular genomic architecture of the region. This indicates that some environmental or genetic factors promote meiotic NAHR at multiple genomic regions.

Ayabe et al. ${ }^{7)}$ and Butler et al. ${ }^{8)}$ studied birth seasonality in PWS patients with chromosome 15 deletion. In these studies, the common peak of birth frequencies was autumn, but the trough seasons differed, as spring and winter, respectively. These results suggested that one or more environmental factors stimulate the development of microdeletions in the autumnborn patients.

In the present study, we investigated seasonal variability caused by both deletion and UPD in Korean patients with PWS, comparing the two groups with the general population.

\section{Materials and methods}

This study protocol was approved by the Institute Review Board Committees at the Samsung Medical Center. A total of 211 patients (129 males, 82 females) born from 1980 to 2014 were diagnosed with PWS at Samsung Medical Center using methylation analysis. All patients met the consensus clinical criteria for PWS. Molecular diagnosis for the deletion group was based on the findings of fluorescence in situ hybridization (FISH) using a probe for the Small nuclear ribonucleoprotein polypeptide $\mathrm{N}$ region at $15 \mathrm{q} 11.2$, and diagnosis for the UPD group was based on the results of microsatellite analysis for several loci on chromosome 15.

We studied differences in the seasonal distribution of births between the deletion group and the Korean general population (Annual Vital Statistics Data from the Statistics Korea website [http://www.kostat.go.kr/] obtained from 6,911,111 individuals born between 2000-2013; data were only available from the year 2000), and between the UPD group and the general population. Because the at-risk population (reference population) was available for the years 2000-2013, 138 patients with PWS who were born from 2000-2013 were compared with the general population.

The birth months of the patients were grouped into four seasons: spring (March-May), summer (June-August), autumn (September-November), and winter (December-February).

In addition, we studied the seasonal distribution of the estimated conception days for 99 patients whose hospital records of gestational age at birth were available. The 14th day after the mother's last menstrual period was considered as the day of estimated conception.

We first analyzed whether there was a nonuniform distribu- tion in the season of birth using the chi-square test. Then, to investigate potential seasonal patterns, we used the Walter and Elwood method9) (R environment version 2.11.1; http://www. r-project.org/) and a cosinor model10) to confirm a sinusoidal (parametric) seasonal pattern of PWS patients. The data were compared with the pattern of total live births from 2000-2013 in Korea $(n=6,911,111)$. Full details of this technique can be found in earlier reports that have used this approach to model the seasonality of childhood leukemia ${ }^{11-14)}$.

In addition, we used Fisher exact test to determine whether there are nonrandom associations between season and PWS group (deletion and UPD) based on the date of conception. In all analyses, a P-value of less than 0.05 was considered to be statistically significant.

\section{Results}

\section{Proportion of genotype characteristics in study subjects}

A total of 211 patients with PWS (birth year: 1980-2014) were diagnosed at Samsung Medical Center. Among them, 122 (57\%) had 15q11.2-q13 microdeletion (deletion group), and 35 patients (17\%) had UPD(15) mat (UPD group). FISH was not performed in the other 52 patients (25\%). Two patients (1\%) were determined to have IC defects (Table 1).

Of the 211 patients with PWS, 138 (65\%) were born from 2000-2013. Among these 138 patients, 74 (54\%) were classified into a deletion group and $22(16 \%)$ into a UPD group. FISH was

Table 1. Proportions of group of patients with Prader-Willi syndrome (PWS)

\begin{tabular}{lccc}
\hline \multirow{2}{*}{ Group } & \multicolumn{3}{c}{ Patients with PWS (birth year) } \\
\cline { 2 - 4 } & $\begin{array}{c}1980-2014 \\
(\mathrm{n}=211)\end{array}$ & $\begin{array}{c}2000-2013 \\
(\mathrm{n}=138)\end{array}$ & $\begin{array}{c}2000-2013 \\
(\mathrm{n}=99)^{\mathrm{a}}\end{array}$ \\
\hline Del & $122(58)$ & $74(53)$ & $53(54)$ \\
UPD & $35(16)$ & $22(16)$ & $53(54)$ \\
Not determined subtype ${ }^{\mathrm{b})}$ & $52(25)$ & $41(31)$ & $32(32)$ \\
Imprinting & $2(1)$ & $1(1)$ & $0(0)$ \\
\hline
\end{tabular}

Values are presented as number (\%).

Del, microdeletion group; UPD, maternal uniparental disomy group.

a) Patients with PWS who were born from 2000-2013 whose hospital records of gestational age at birth were available (total 99 patients). ${ }^{b)}$ Patients with PWS were diagnosed by methylation PCR, but did not undergoe fluorescence in situ hybridization.

Table 2. Monthly variation of the Prader-Willi syndrome (PWS) group compared with the at-risk population ${ }^{\text {a) }}$

\begin{tabular}{lcc}
\hline Group & Test statistics $^{\text {b) }}$ & $P$-value \\
\hline Del & 6.04 & $0.0489^{*}$ \\
UPD & 3.39 & 0.1836 \\
Total & 0.96 & 0.6189 \\
\hline
\end{tabular}

Del, microdeletion group; UPD, maternal uniparental disomy group; Total, total patients with PWS.

${ }^{a}$ At-risk population indicates total live births during 2000-2013 in

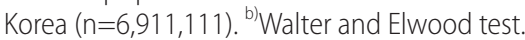

${ }^{*} P<0.05$, statistically significant difference. 


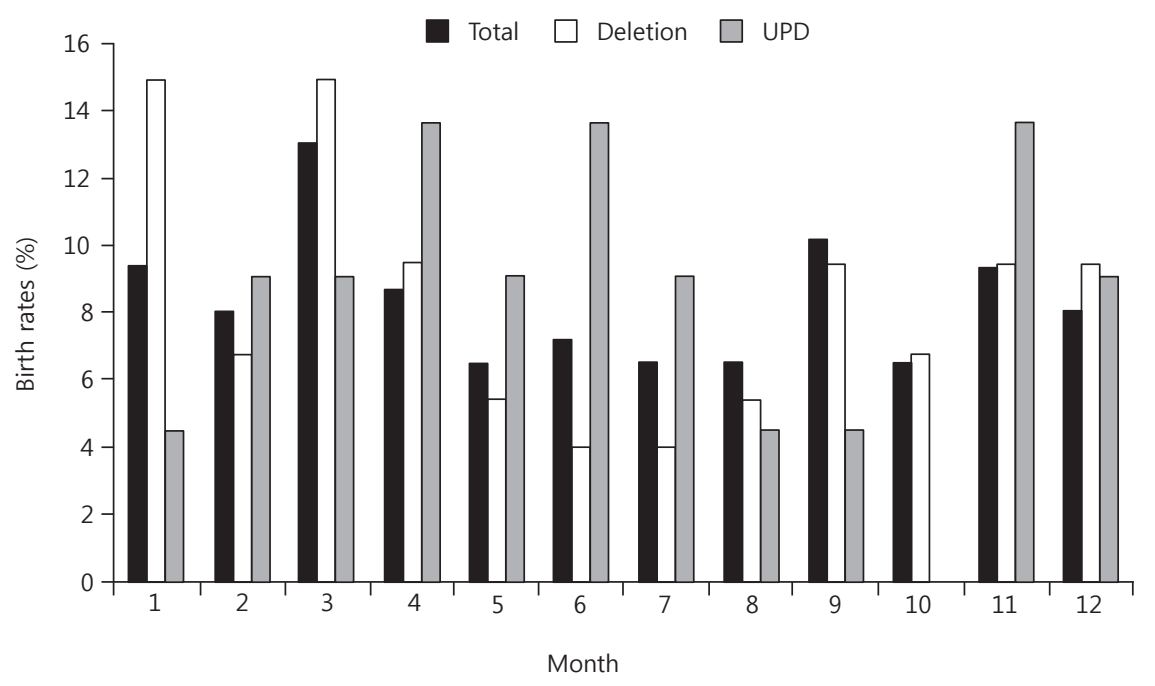

Fig. 1. Monthly distribution of births of patients with Prader-Willi syndrome. In the total group of 138 patients, March was the peak month, with 18 patients (13\%). In the deletion group, birth rate was highest in January and March; each month had 11 patients (14.86\%). In contrast, the lowest birth rate was seen in June and July (3 patients each; 4\%). In the UPD group, there was no apparent systematic distribution. Total, total patients with PWS; Del, microdeletion group; UPD, maternal uniparental disomy group.

Table 3. Cosinor model of patients with Prader-Willi syndrome (PWS) to confirm sinusoidal (parametric) seasonal pattern

\begin{tabular}{lcccc}
\hline Group & Amplitude & $\begin{array}{c}\text { Peak phase } \\
(\mathrm{mo})\end{array}$ & $\begin{array}{c}\text { Low phase } \\
(\mathrm{mo})\end{array}$ & $\begin{array}{c}\text { Significant } \\
\text { seasonality }\end{array}$ \\
\hline Del & 0.23 & 1.2 & 7.2 & TRUE $^{\text {a) }}$ \\
UPD & 0.06 & 4.0 & 10.0 & FALSE \\
Total & 0.14 & 1.6 & 7.6 & FALSE \\
\hline
\end{tabular}

a) Deletion group in the cosinor model was presumed to be significant because peak (maximum) month of cosine curve is January (month, 1.2), whereas low phase (minimum) is July (month, 7.2).

Del, microdeletion group; UPD, maternal uniparental disomy group; Total, total patients with PWS.

not performed in 41 patients out of the 138 (29\%). IC defects were identified in one patient (1\%) (Table 1). The 74 patients in the deletion group and the 22 patients in the UPD group were compared with the at-risk general population.

\section{Monthly and seasonal distributions of birth dates}

To assess whether month of birth influences susceptibility to PWS disorders, we initially compared the distribution of all patients with PWS who were born in 2000 or later with that of the general population. The observed frequencies of birth month in patients with PWS by month and season are shown in Figs. 1 and 2, respectively. There was no statistical significance in seasonal variation in births over the complete PWS population $\left(\mathrm{X}^{2}=7.2522, P=0.2982\right)$.

However, statistical significance was found in the monthly variation of the PWS deletion group compared with the at-risk population $(P=0.0489)$ (Table 2$)$. For the UPD group, birth dates

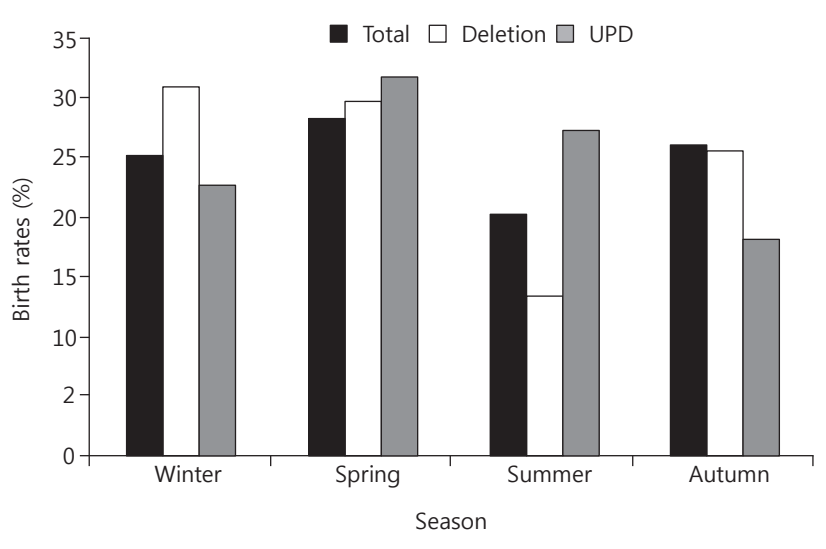

Fig. 2. Seasonal distribution of births of patients with Prader-Willi syndrome (PWS). The peak season of PWS in the deletion group was winter,with 23 patients (31\%); summer was the lowest, with 10 patients (13\%). For the total of 138 patients with PWS (birth year: 2000-2013), spring was a peak season with 39 patients (28\%), and summer was the lowest, with 28 patients (20\%). In the uniparental disomy (UPD) group, peak season was spring, with seven patients (31\%), and trough season was autumn, with four patients (18\%). The relationship between the PWS group (deletion, mUPD, total) and seasonality was not statistically significant (chi-square test, $P=0.2982$ ). Spring (March-May), summer (June-August), autumn (September-November), winter (DecemberFebruary). Total, total patients with PWS; Del, microdeletion group; UPD, maternal uniparental disomy group.

occurred most frequently in spring, especially in April, but this result was not statistically significant $\left(\mathrm{X}^{2}=3.39, P=0.1836\right)$.

Based on these results, we applied the cosinor model to patients with PWS (Table 3). The peak birth month for PWS patients with deletion was January, while the nadir occurred in July. This result was statistically significant (amplitude $=0.23$, phase $=1.2$, low point $=7.2$ ). In contrast, neither the total PWS 
group nor the mUPD group results were statistically significance when analyzed with the cosinor model.

\section{Monthly and seasonal distributions of estimated conception dates}

We studied the seasonal distribution of the estimated conception days for 99 patients who were born from 2000 to 2013 whose hospital records of gestational age at birth were available (The mean \pm standard deviation gestational age was $38.8 \pm 2.0$ weeks).

Of the 99 patients, 53 (54\%) were classified into a deletion group and 14 (14\%) into a UPD group. FISH was not performed in 32 patients (32\%). No patients with IC defects were identified (Table 1). Because no data related to the estimated conception day were available for the general population, we analyzed the 53 patients in the deletion group and the 14 patients in the UPD group.

Deletion group incidence was much higher in spring and lower in autumn, compared with the other seasons. These findings were statistically significant (Fisher exact test, $P=0.0168$ ) (Supplementary Fig. 1A).

On a monthly basis, April and May were the highest months of conception, whereas October and February were the lowest (Supplementary Fig. 1B).

\section{Discussion}

This is the first study addressing correlations of seasonal variability with Korean PWS patients. In this study, a clear seasonal birth distribution was observed, with a peak in winter and a trough in summer in the PWS deletion group compared with the general population. Birth distribution was lowest in July and peaked in January. The distribution of UPD group births was not seasonal.

Studying the pathogenesis of deletion on chromosome 15 is important because the deletion group accounts for $70 \%$ of the PWS population. Our results differ from those of previous studies addressing the seasonal variability of the PWS populations with deletion in the United States (US) and Japan ${ }^{7,8)}$. The peak season of the deletion group was autumn in the US and Japan, but winter in our study. The study in Japan had used only Walter and Elwood's test statistics, but we additionally employed cosinor analysis to confirm sinusoidal seasonal pattern. The US study had analyzed only a 61 patients in the deletion group without comparison with the general population; in contrast, we compared PWS patients with the general population. Nevertheless, differences in the various prevalence rates of the PWS deletion might be related to differences in ethnicity, nation, and environmental factors present in these various studies.

Several hypotheses about the pathogenesis of the PWS deletion group suggest some supportive clues. PWS is a genomic disorder caused by meiotic chromosomal rearrangements of unstable regions flanked by Low Copy Repeats (LCRs) ${ }^{15)}$. The 15q11-q13 region is flanked by three LCRs (LCR15-1, LCR152, and LCR15-3) in which most of the deletion breakpoints in a PWS case occur ${ }^{16)}$. The 15q11-q13 LCRs called END-repeats act as hot-spots that could predispose the particular genomic architecture of the $15 \mathrm{q} 11-\mathrm{q} 13$ region to $\mathrm{NAHR}^{6,17)}$. The interchromatid NAHR is the predominant mechanism involved in the generation of sperm deletions and duplications ${ }^{15)}$. The rate of sperm deletion and duplication in $15 \mathrm{q} 11-\mathrm{q} 13$ is considered relevant to particular haplotypes and interchromatid exchange events ${ }^{15)}$. In particular, a significant percentage of PWS fathers have an increased susceptibility to generate 15q11q13 deletions in spermatozoa, suggesting the participation of intrachromatid NAHR exchanges ${ }^{6}$. NAHR plays a central role during meiosis and recombination. Furthermore, frequency at hotspots is sensitive to environmental conditions, such as high temperature, nitrogen starvation, ultraviolet (UV) radiation, oxidative stress, and high osmolarity ${ }^{18)}$.

In addition, homologous recombination (HR) is an important pathway in mammalian cells as both a pathway for DNA repair and a cause for genomic rearrangements affected by UV radiation ${ }^{19,20)}$. Bishop and Schiestl ${ }^{19)}$ suggested that "correct" HR initiated by appropriate UV radiation can repair DNA damage, in contrast to "aberrant" HR, which can cause genomic rearrangements such as deletion. In this regard, the presence of vitamin $\mathrm{D}$ receptors in human sperm ${ }^{21)}$ and the relationship between vitamin D levels reflecting the UV radiation and sperm parameters $^{22,23)}$ are interesting. Hammoud et al. ${ }^{23)}$ found that low vitamin D levels were correlated with lower total sperm count and reduced sperm motility.

A study on the analysis of vitamin D levels of Koreans, based on The Korea National Health and Nutrition Examination Survey 2008, is relevant to this discussion. This study assessed 2,504 men over the age of 20. Vitamin D level was the lowest in men aged from 20-29 years. Mean serum $25(\mathrm{OH}) \mathrm{D}$ level was the lowest in spring $(17.8 \mathrm{ng} / \mathrm{mL})$, followed by in winter $(18.4$ $\mathrm{ng} / \mathrm{mL}$ ). In addition, the summer season was the highest $(24.4$ $\mathrm{ng} / \mathrm{mL})$, followed by autumn $(23.9 \mathrm{ng} / \mathrm{mL})^{24)}$.

Considering that spermatogenesis occurs at approximately the same time as the conception day, and that the gestational age of most of the PWS with deletion patients in our study was near full term, their season of conception was spring. This is consistent with our results as shown in supplementary Fig. 1A, B.

Based on the finding that the season with the lowest vitamin D level and the peak season of conception of the Korean PWS deletion group are the same (i.e., both are spring), we can suggest a new hypothesis that NAHR-induced deletion through spermatogenesis defects from vitamin D insufficiency occur most often in spring. In other words, it is expected that the vitamin D level of a PWS father might be related to a defect of spermatogenesis, including copy number abnormalities resulting in microdeletion with PWS. However, we need to examine the relationship between vitamin $\mathrm{D}$ level and microdeletion in PWS through the further study of vitamin D level status in PWS fathers.

The present study has some limitations. Statistical analysis was 
not performed for the seasonality of the estimated conception day because of the lack of Korean reference data for comparison. However, in contrast to previous studies that evaluated the seasonal variability of patients with PWS, we applied not only the Walter and Elwood method that is commonly used statistical analysis of seasonal variation, but also applied the cosinor test, which compensates for limitations in the Walter and Elwood method. This use of two analysis methods imparts high reliability to our results.

In conclusion, we have investigated seasonal distribution of the PWS population in Korea. The information reported here may serve as a helpful baseline study for future work relating PWS patients with deletion to environmental factors, such as vitamin D level. Further study of the correlation between the deletion group of the PWS population and seasonal distribution will be required to provide more useful information about detection and prevention of PWS.

\section{Conflict of interest}

No potential conflict of interest relevant to this article was reported.

\section{Acknowledgments}

This study was supported by a grant from Samsung Medical Center (\#GFO1130061).

\section{Supplementary material}

Supplementary Fig. 1 can be found via http://www.e-apem. $\mathrm{org} / \mathrm{src} / \mathrm{sm} / \mathrm{apem}-20-40$-s001.pdf.

\section{References}

1. Bittel DC, Butler MG. Prader-Willi syndrome: clinical genetics, cytogenetics and molecular biology. Expert Rev Mol Med 2005;7:1-20.

2. Yazdi PG, Su H, Ghimbovschi S, Fan W, Coskun PE, Nalbandian A, et al. Differential gene expression reveals mitochondrial dysfunction in an imprinting center deletion mouse model of Prader-Willi syndrome. Clin Transl Sci 2013;6:347-55.

3. Nicholls RD, Knoll JH, Butler MG, Karam S, Lalande M. Genetic imprinting suggested by maternal heterodisomy in nondeletion Prader-Willi syndrome. Nature 1989;342:2815.

4. Ledbetter DH, Riccardi VM, Airhart SD, Strobel RJ, Keenan BS, Crawford JD. Deletions of chromosome 15 as a cause of the Prader-Willi syndrome. N Engl J Med 1981;304:325-9.

5. Cassidy SB, Driscoll DJ. Prader-Willi syndrome. Eur J Hum Genet 2009;17:3-13.

6. Molina O, Blanco J, Vidal F. Deletions and duplications of the 15q11-q13 region in spermatozoa from Prader-Willi syndrome fathers. Mol Hum Reprod 2010;16:320-8.
7. Ayabe T, Matsubara K, Ogata T, Ayabe A, Murakami N, Nagai T, et al. Birth seasonality in Prader-Willi syndrome resulting from chromosome 15 microdeletion. Am J Med Genet A 2013;161A:1495-7.

8. Butler MG, Ledbetter DH, Mascarello JT. Birth seasonality in Prader-Willi syndrome. Lancet 1985;2:828-9.

9. Walter SD, Elwood JM. A test for seasonality of events with a variable population at risk. Br J Prev Soc Med 1975;29:1821.

10. Bingham C, Arbogast B, Guillaume GC, Lee JK, Halberg F. Inferential statistical methods for estimating and comparing cosinor parameters. Chronobiologia 1982;9:397-439.

11. Harris RE, Harrell FE Jr, Patil KD, Al-Rashid R. The seasonal risk of pediatric/juvenile acute lymphocytic leukemia in the United States. J Chronic Dis 1987;40:915-23.

12. Meltzer AA, Annegers JF, Spitz MR. Month-of-birth and incidence of acute lymphoblastic leukemia in children. Leuk Lymphoma 1996;23:85-92.

13. Westerbeek RM, Blair V, Eden OB, Kelsey AM, Stevens RF, Will AM, et al. Seasonal variations in the onset of childhood leukaemia and lymphoma. Br J Cancer 1998;78:119-24.

14. Douglas S, Cortina-Borja M, Cartwright R. A quest for seasonality in presentation of leukaemia and nonHodgkin's lymphoma. Leuk Lymphoma 1999;32:523-32.

15. Molina O, Anton E, Vidal F, Blanco J. Sperm rates of $7 \mathrm{q} 11.23,15 \mathrm{q} 11 \mathrm{q} 13$ and $22 \mathrm{q} 11.2$ deletions and duplications: a FISH approach. Hum Genet 2011;129:35-44.

16. Amos-Landgraf JM, Ji Y, Gottlieb W, Depinet T, Wandstrat AE, Cassidy SB, et al. Chromosome breakage in the PraderWilli and Angelman syndromes involves recombination between large, transcribed repeats at proximal and distal breakpoints. Am J Hum Genet 1999;65:370-86.

17. Emanuel BS, Shaikh TH. Segmental duplications: an 'expanding' role in genomic instability and disease. Nat Rev Genet 2001;2:791-800.

18. Koren A, Ben-Aroya S, Kupiec M. Control of meiotic recombination initiation: a role for the environment? Curr Genet 2002;42:129-39.

19. Bishop AJ, Schiestl RH. Homologous recombination as a mechanism for genome rearrangements: environmental and genetic effects. Hum Mol Genet 2000;9:2427-334.

20. Covo S, Ma W, Westmoreland JW, Gordenin DA, Resnick MA. Understanding the origins of UV-induced recombination through manipulation of sister chromatid cohesion. Cell Cycle 2012;11:3937-44.

21. Corbett ST, Hill O, Nangia AK. Vitamin D receptor found in human sperm. Urology 2006;68:1345-9.

22. Blomberg Jensen M, Bjerrum PJ, Jessen TE, Nielsen JE, Joensen UN, Olesen IA, et al. Vitamin D is positively associated with sperm motility and increases intracellular calcium in human spermatozoa. Hum Reprod 2011;26: 1307-17.

23. Hammoud AO, Meikle AW, Peterson CM, Stanford J, Gibson M, Carrell DT. Association of 25-hydroxy-vitamin $\mathrm{D}$ levels with semen and hormonal parameters. Asian J 
Androl 2012;14:855-9.

24. Choi HS, Oh HJ, Choi H, Choi WH, Kim JG, Kim KM, et al. Vitamin D insufficiency in Korea: a greater threat to younger generation: the Korea National Health and Nutrition Examination Survey (KNHANES) 2008. J Clin Endocrinol Metab 2011;96:643-51. 